\title{
Numerical study of inelastic buckling behavior of rectangular steel plates with circular openings under shear forces
}

\author{
Rajawali M Akbar ${ }^{1}$ and Bambang Suryoatmono ${ }^{1, *}$ \\ ${ }^{1}$ Department of Civil Engineering, Faculty of Engineering, Parahyangan Catholic University, Bandung, Indonesia
}

\begin{abstract}
Cellular steel beam is flanged steel beam with circular openings of uniform diameter and distance between each opening. The main benefit of such beam is to reduce the structural weight without reducing the strength significantly. A rectangular steel plate with circular opening is frequently used as a model of a web panel of such beam with vertical web stiffeners. The dimension of the plate is the dimension of the web bounded by top and bottom flanges and two adjacent vertical stiffeners. In this research, finite element method is utilized to perform inelastic buckling analyses of rectangular steel plates with circular openings under shear forces along all four edges assuming steel as elastic-perfectly-plastic material with yield stress of $250 \mathrm{MPa}$. Both nonlinear geometry and nonlinear material are considered in the analyses. The objective of this research is to study buckling behavior of the plate in terms of buckling mode, critical load, and Von Mises (effective) stress distribution. The buckling shear loads of the plates of various length-to-width ratios of the plate (1.0, 1.25 , and 1.50$)$ and various opening-diameter-to-plate-width ratios $(0.00,0.05,0.10,0.15,0.20,0.25$, $0.30,0.35,0.40,0.45,0.50$ ) have been obtained from the analyses. The deformation and Von Mises stress distribution at every load level have been obtained as well from the finite element analyses. Equation to predict inelastic buckling shear force of a rectangular steel plates with circular opening under shear forces is proposed in this study. Verification of the method has been performed by comparing shear buckling loads resulted from finite element analyses with the analytical results in the elastic range.
\end{abstract}

\section{Introduction}

The use of cellular steel beams is frequently inevitable in structural systems because the presence of circular openings in such beams are required for mechanical and electrical systems or to reduce the weight of the structural systems. When the web of such beams experience compression loads, bending moments, or shear forces, they may show instability or buckle due to principal compression stresses. To increase the strength and stiffness of such webs, vertical web stiffeners are often used. In analyzing such webs, it is generally acceptable to assume the webs as plates with the dimension bounded by top and bottom flanges and two adjacent vertical stiffeners.

The dimension of a web panel or a plate is $a \times h$ as seen in Fig, 1. If a web panel of thickness $t$ is subjected to shear force, then the differential element of the panel experiences tension and compression principal stresses as seen in the figure. If the plate is too slender, i.e. the ratio of $h / t$ is too large, then buckling occurs. If all stresses in the plate is in the elastic range during buckling, then it is called elastic shear buckling. On the other hand, if some or all stresses in the plate is beyond the elastic range, then it is called inelastic shear buckling.
Several studies have been conducted on buckling of plates. By employing finite element method (FEM), Real et al. [1] evaluated both elastic and inelastic buckling loads of uniaxially loaded rectangular plates with circular openings. The results show that while the circular opening can in some cases even increase the elastic buckling load, the inelastic buckling load is reduced by the presence of the openings. Maiorana et al. [2] conducted elastic buckling analyses of rectangular and square plates with rectangular and circular openings in various positions subjected to combined axial compression and bending moment. The aim was to give some practical indications on the best position and orientation of rectangular openings and the best position of the circular opening in steel plates under combined axial compression and bending moment. FEM is also used in study of buckling of rectangular plates with openings subjected to various in-plane compressive edge loading [3]. While in cellular beam, the web is prone to shear buckling, the three studies mentioned above were not focused on shear buckling.

The elastic shear buckling analyses of tapered plates with circular opening under shear stress has been conducted numerically employing FEM [4]. Several parameters are considered in the study, namely aspect ratios, tapering ratios and circular opening diameters. Results show that the small opening diameter has no

* Corresponding author: suryoatm@unpar.ac.id 
significant effect on the critical shear loads. Based on elastic and inelastic analyses of steel plates with circular and rectangular openings under shear loading, Pellegrino concludes that opening dimensions have strong influence on the shear buckling load [5]. Recently, Pham utilized Spline Finite Strip Method (SFSM) to study elastic shear buckling firstly for the square plates with openings and subsequently for the whole thin-walled lipped channel sections with centrally located openings [6].

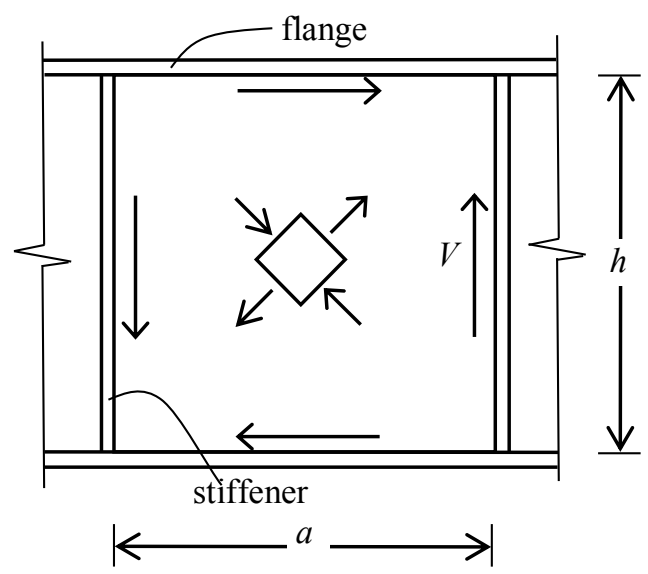

Fig. 1. Diagonal tension and compression principal stresses in a web panel.

By employing FEM and assuming elastic behavior of the material, Moen and Schafer analyze thin plates with openings in compression or bending, and conclude that openings may create unique buckling modes, and can either increase or decrease elastic buckling stress depending on the opening geometry and spacing [7].

In this research, rectangular plates with centrally located circular openings under shear forces as seen in Fig. 2 are analyzed by utilizing FEM and assuming that steel is elastic-perfectly-plastic material. The objective of this research is to study buckling behavior of the plate in terms of buckling mode, critical load, and Von Mises stress distribution. The parameters considered are the ratio between opening diameter $d_{h}$ and plate width $h$ and the ratio between the plate length $a$ and width $h$. Equation to predict buckling loads of rectangular steel plates with centrally located circular openings under shear forces are proposed in this study.

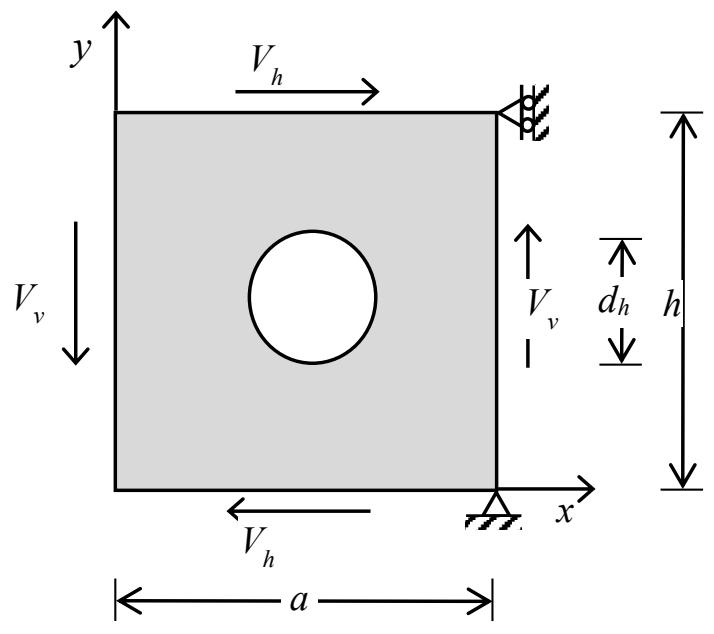

Fig. 2. A plate with centrally located circular opening under shear forces.

\section{Method}

\subsection{Finite element modeling}

The commercially available ANSYS software is utilized in this study. The element type used is shell (SHELL181) with 4 nodes per element. Each node has 6 degrees of freedom (three displacements in $x, y$, and $z$ directions, and three rotations about $x, y$, and $z$-axes). To prevent rigid body motion, the in-plane boundary conditions are applied as shown in Fig. 2, i.e. hinge at bottom right corner and roller at right top corner of the plate. Out-ofplane displacements ( $z$-direction displacements) of all four edges are restrained. Although all elements, nodes, and loads are in $x, y$ plane, in buckling analyses the outof-plane displacements of all nodes other than the ones at the edges are not restrained.

Two types of analyses are carried out, namely eigenvalue buckling analysis and inelastic nonlinear buckling analysis. Large deformation theory applies in the analyses.

The horizontal and vertical shear loads $\left(V_{h}\right.$ and $V_{v}$ in Fig. 2) are distributed evenly on each plate edge. In eigenvalue buckling analysis, these loads are arbitrarily taken as $10 \mathrm{~N} / \mathrm{mm}$. In inelastic buckling analysis, these are increasing loads from zero until failure occurs indicated by nearly zero determinant of structural stiffness matrix.

Web height $h$ (= plate width) is kept constant of 500 $\mathrm{mm}$. Plate length $a$ varies according to length-to-width ratio of $1.00,1.25$, and 1.50 . Plate thickness is $6 \mathrm{~mm}$. Opening-diameter-to-width ratio varies from 0.00 to 0.500 with an increment of 0.05 .

Steel is modeled as elastic-perfectly-plastic material. Stress-strain relationship for such material is shown in Fig. 3. The same relationship is used for tension and compression. Yield stress $F_{y}$ is $250 \mathrm{MPa}$ and modulus of elasticity is $200 \mathrm{GPa}$. Poisson's ratio of 0.30 is used. As commonly used for ductile material like steel, Von Mises failure criterion is used.

The automatic mesh generation feature of the software is utilized. Typical finite element mesh is shown in Fig. 4. 
As seen in the figure, the elements size in the vicinity of the opening are small enabling to capture large stress gradient in the area.

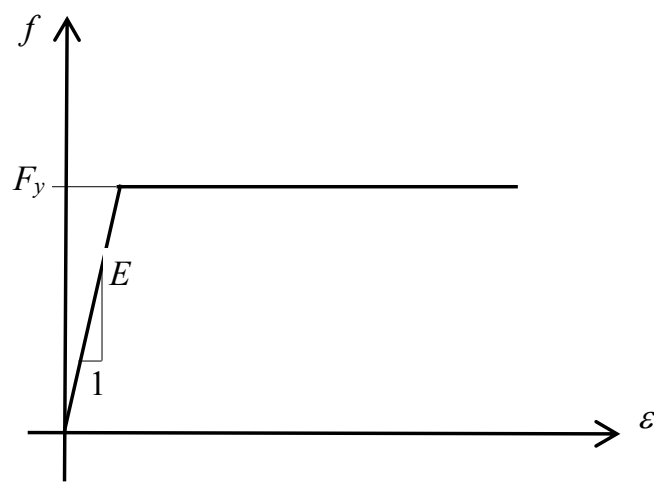

Fig. 3. Stress - strain relationship of an elastic-perfectly-plastic material.

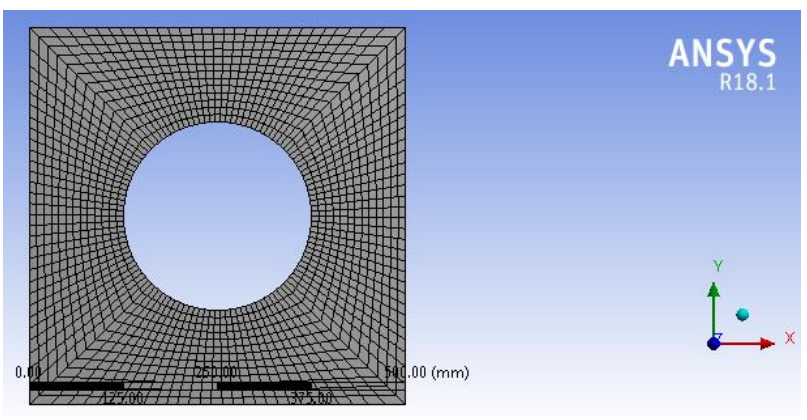

Fig. 4. Typical finite element mesh.

\subsection{Elastic eigenvalue buckling analysis}

Elastic eigenvalue buckling analysis is an analysis that incorporates second order effects in structural stiffness matrix. Buckling loads and buckling deformations obtained from the analysis by theoretically making the determinant of the matrix equals to zero, are the eigenvalues and eigenvectors, respectively. In this study, elastic buckling analysis is performed for two tasks. The first task is to verify the FEM by comparing the results with the available analytical results. The second task is to establish the first buckling mode that is needed as initial condition in the inelastic buckling analysis. For example, Fig. 5 shows out-of-plane $(z$ direction $)$ relative displacement of first buckling mode obtained from elastic eigenvalue buckling analysis for $a / h=1.0$ and $d_{h} / h=0.5$.

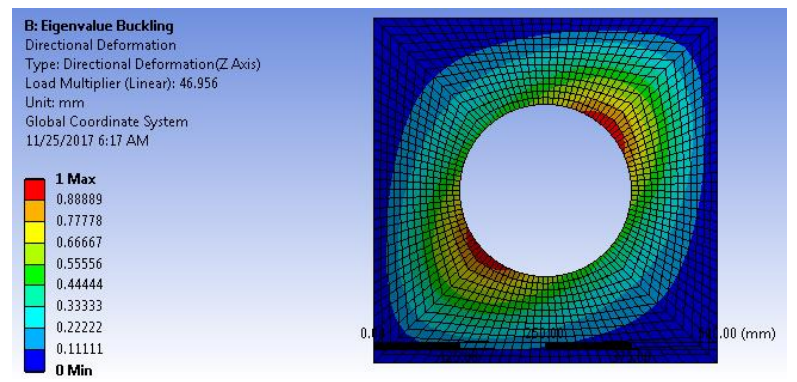

Fig. 5. Out-of-plane relative displacement of first buckling mode obtained from elastic eigenvalue buckling analysis for $a / h=1.0$ and $d_{h} / h=0.5$.
In ANSYS software, the shear force is input as uniformly distributed load. Then the critical or buckling shear force $V_{c r}$ is computed by using $V_{c r}=$ (given distributed load) $\times \lambda \times$ t, where $\lambda$ is load multiplier obtained from eigenvalue buckling analysis.

For a rectangular plate of the size $a \mathrm{x} h$ with thickness $t$ under in-plane shear force $V$, the analytical buckling shear force $V_{c r}$ is $V_{c r}=\tau_{c r} h t$. The buckling shear stress $\tau_{c r}$ as developed by Timoshenko and Woinowski-Krieger [8] is

$$
\begin{gathered}
\tau_{c r}=k \frac{\pi^{2} E}{12\left(1-v^{2}\right)}\left(\frac{t}{h}\right)^{2} \\
k=5.34+\frac{4}{(a / h)^{2}} \quad \text { for } a / h>1 \\
k=4+\frac{5.34}{(a / h)^{2}} \quad \text { for } \quad a / h \leq 1
\end{gathered}
$$

Comparison between buckling shear force $V_{c r}$ obtained from analytical equation (1) and FEM is shown in Table 1. It is clear that FEM is very accurate in predicting elastic buckling shear force.

Table 1. Comparison between elastic buckling shear load $V_{c r}$ for plate without opening obtained from analytical equation and FEM.

\begin{tabular}{|c|c|c|c|}
\hline \multirow{2}{*}{$a / h$} & \multicolumn{2}{|c|}{$V_{c r}(\mathrm{kN})$} & \multirow{2}{*}{$\begin{array}{c}\text { Difference } \\
(\%)\end{array}$} \\
\cline { 2 - 3 } & Analytical & FEM & -0.56 \\
\hline 1.00 & 243.1 & 241.8 & -2.00 \\
\hline 1.25 & 205.6 & 201.5 & -0.89 \\
\hline 1.50 & 185.3 & 183.6 & \\
\hline
\end{tabular}

\subsection{Inelastic buckling analysis}

In inelastic buckling analysis, step-by-step integration is used. Incremental in-plane shear force is applied to the plate. If in-plane shear force were applied to perfect undeformed plate, then out-of-plane deformation would not occur. Therefore, some initial imperfection that includes out-of-plane deformation has to be applied. The first buckling mode obtained from elastic eigenvalue buckling analysis is used as the initial deformation in the inelastic buckling analysis. In ANSYS, this task is performed through insertion of special UPGEOM commands. Since buckling mode is relative value of a structural deformation, an arbitrary small amplitude of $\frac{\sqrt{h a}}{1500}$ is chosen in this study. It is within the range suggested by Ahn et al. [9], i.e. between $h / 10,000$ and $h / 100$. The latter is the maximum plate imperfection according to Bridge Welding Code [10]. In this study $\sqrt{h a}$ is used as a replacement of $h$ to reflect the fact that the plate is rectangular not square, especially for $a / h=1.25$ and 1.50 .

As mentioned before, incremental in-plane shear forces are applied to the plate in the directions as seen in Fig. 2. Boundary conditions are also shown in the figure. Stress-strain relationship as seen in Fig. 3 is entered through Structural Steel NL in ANSYS. 


\section{Results and discussions}

One of the results of inelastic buckling analysis is the inplane shear force versus out-of-plane deflection curve as seen in Fig. 6, 7, and 8 for $a / h=1.00,1.25$, and 1.50 respectively. The peak of each curve is taken as the inelastic buckling shear force. Obviously, the critical shear force $V_{c r}$ is smaller for larger $d_{h} / h$ ratio. This conclusion applies for all $a / h$ values investigated in this study. Furthermore, it can be seen that at any given shear force, the out-of-plane displacement is larger for larger opening in the plate. Another observation from the figures is that for any $d_{h} / h$ ratio, the largest buckling shear force is for aspect ratio $a / h=1.5$ and the smallest one is for $a / h$ $=1.0$. The difference, however, is quite small.

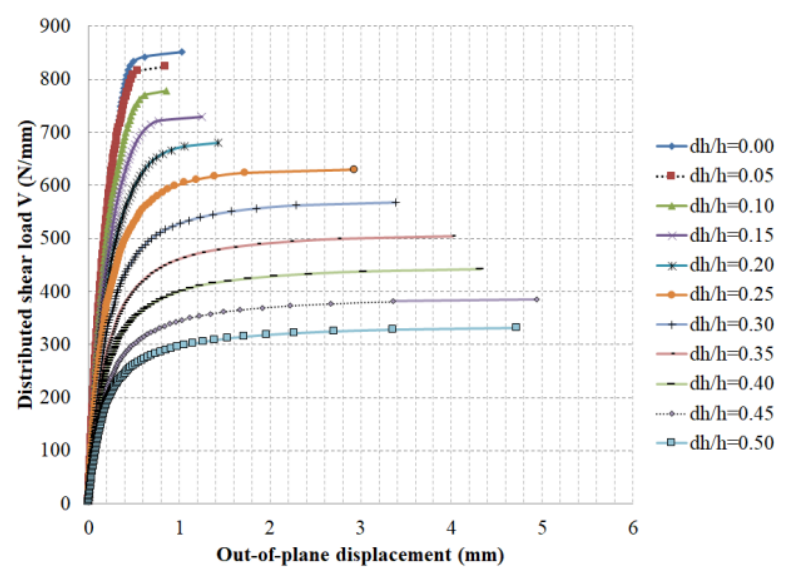

Fig. 6. Distributed shear force $V(\mathrm{~N} / \mathrm{mm})$ versus out-of-plane displacement $(\mathrm{mm})$ for $a / h=1$ and various $d_{h} / h$.

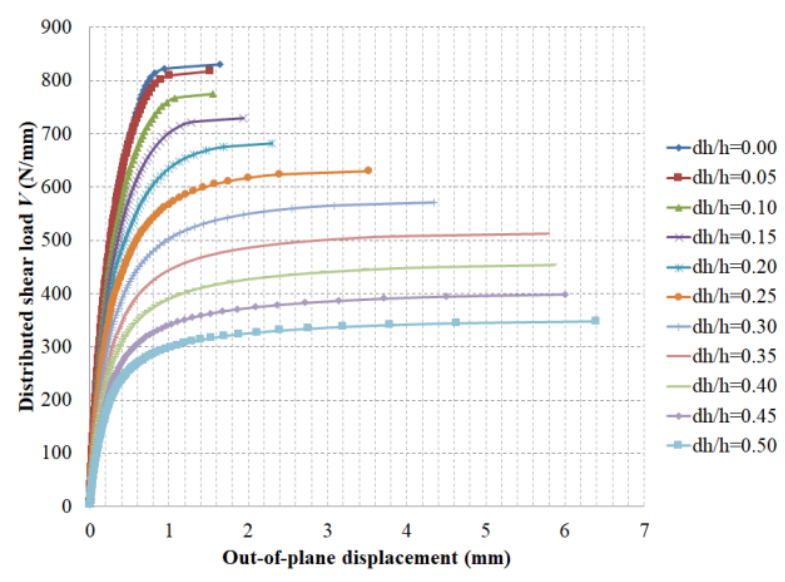

Fig. 7. Distributed shear force $V(\mathrm{~N} / \mathrm{mm})$ versus out-of-plane displacement (mm) for $a / h=1.25$ and various $d_{h} / h$.

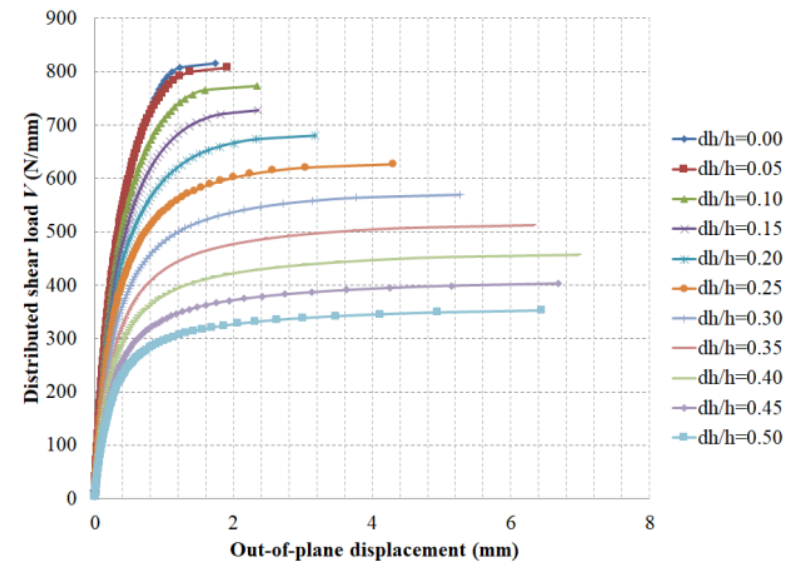

Fig. 8. Distributed shear force $V(\mathrm{~N} / \mathrm{mm})$ versus out-of-plane displacement $(\mathrm{mm})$ for $a / h=1.5$ and various $d_{h} / h$.

Another interesting result of inelastic buckling analysis is Von Mises stress distribution at any given shear load level. Fig. 9, 10, 11, and 12 show Von Mises stress distribution obtained from inelastic buckling analysis for $a / h=1.0$ and $d_{h} / h=0.5$ loaded at $25 \%, 50 \%$, $75 \%$, and $100 \%$ of buckling shear force $V_{c r}$. Observe that even at shear force level below $25 \%$ of shear buckling force, yielding has started. Clearly, yielding starts at certain parts of the perimeter of the opening and spreads towards the plate edge as the shear force is increased. Furthermore, at buckling shear force level, there is some area in the plate that is still in the elastic range.

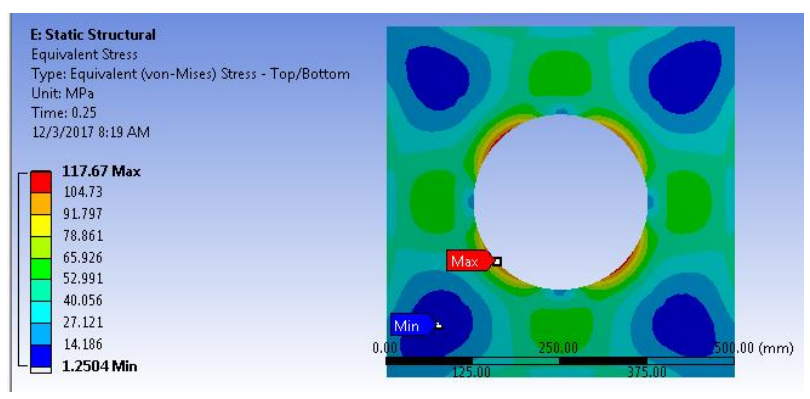

Fig. 9. Von Mises stress distribution obtained from inelastic buckling analysis for $a / h=1.0$ and $d_{h} / h=0.5$ loaded at $25 \%$ of inelastic buckling shear force $V_{c r}$.

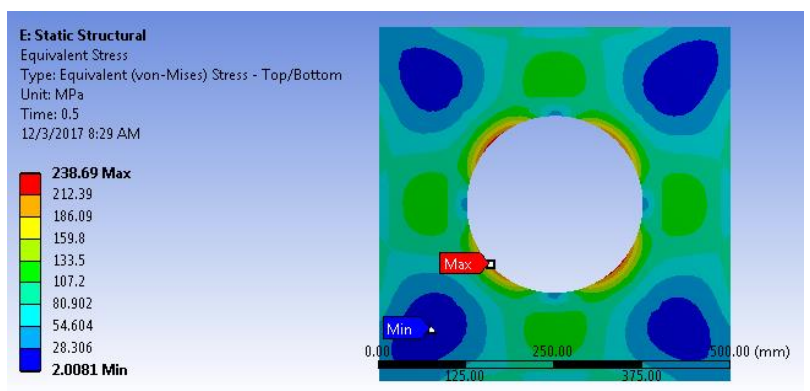

Fig. 10. Von Mises stress distribution obtained from inelastic buckling analysis for $a / h=1.0$ and $d_{h} / h=0.5$ loaded at $50 \%$ of inelastic buckling shear force $V_{c r}$. 


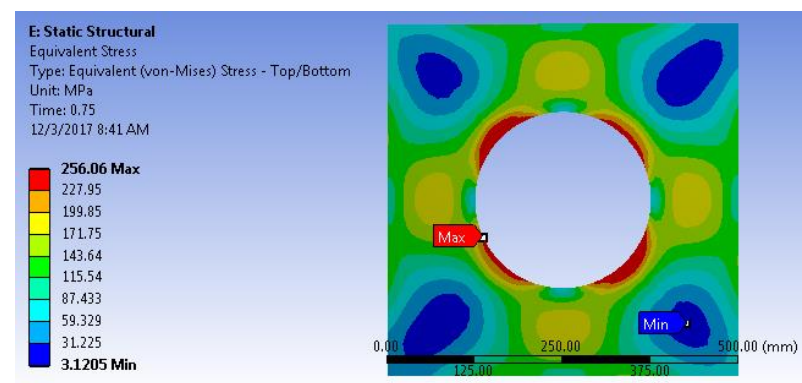

Fig. 11. Von Mises stress distribution obtained from inelastic buckling analysis for $a / h=1.0$ and $d_{h} / h=0.5$ loaded at $75 \%$ of inelastic buckling shear force $V_{c r}$.

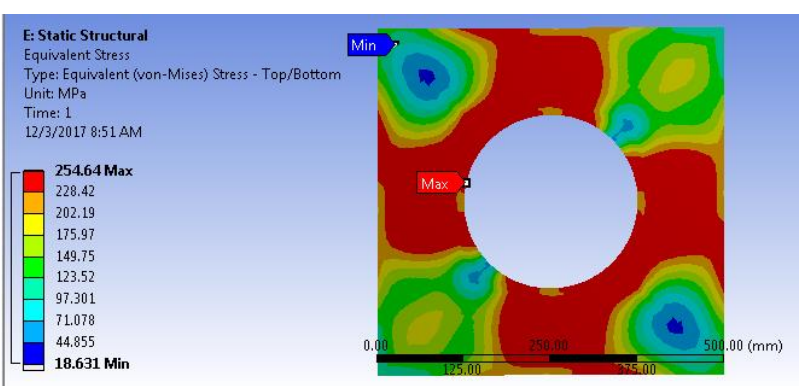

Fig. 12. Von Mises stress distribution obtained from inelastic buckling analysis for $a / h=1.0$ and $d_{h} / h=0.5$ loaded at inelastic buckling shear force $V_{c r}$.

To see the influence of opening diameter on the buckling shear force, Fig. 13 shows inelastic $V_{c r} /\left(V_{c r}\right)_{\text {no hole }}$ versus $d_{h} / h$ for various $a / h$, where $\left(V_{c r}\right)_{\text {no hole }}$ is inelastic buckling shear force for the same plate without opening. It is seen that the relationship between the two variables is nearly linear for all aspect ratios in this study.

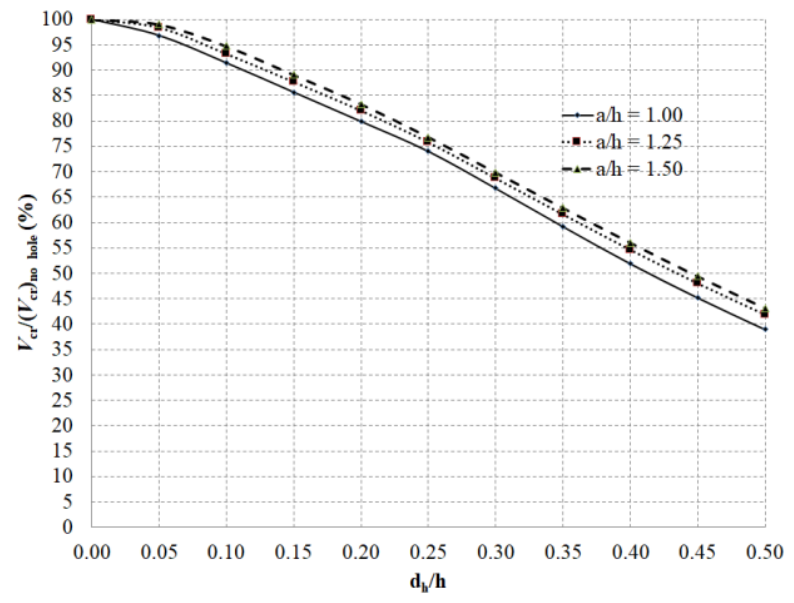

Fig. 13. Inelastic $V_{c r} /\left(V_{c r}\right)_{n o}$ hole versus $d_{h} / h$ for various $a / h$.

If the ratio between inelastic buckling shear force $V_{c r \text {,inelastic }}$ and elastic shear buckling load $V_{c r, e l a s t i c}$ is plotted versus $d_{h} / h$ for various $a / h$ as seen in Fig. 14, it is seen that the relationship between the two variables is nearly linear for all aspect ratios in this study, although the linear relationships is not as strong as the ones in Fig. 13.

The data used to plot Fig. 13 and 14 is shown in Table 2. To find equation that can be used to predict inelastic buckling shear force of a rectangular plate with opening, multiple regression is performed for the data shown in the table. If $d_{h} / h$ is denoted by $x_{1}, a / h$ is denoted by $x_{2}$ and $V_{c r} /\left(V_{c r}\right)_{\text {no hole }}$ is denoted by $y$ (in $\left.\mathrm{kN}\right)$, then the regression equation is

$$
y=-0.826 x_{1}+0.267 x_{2}+0.367
$$

with coefficient of determination $r^{2}=0.983$. The coefficient is very close to 1.0 indicating that Eq. 4 is very good to predict $y$ for any given values of $x_{1}$ and $x_{2}$. More clearly, inelastic buckling shear force of a rectangular plate with opening can be predicted by using

$$
V_{c r}=\left[-0.826\left(\frac{d_{h}}{h}\right)+0.267\left(\frac{a}{h}\right)+0.367\right] \tau_{c r} h t
$$

where $\tau_{c r}$ is elastic buckling shear stress for the same plate without opening computed using Eq. 1. It should be noted that the proposed equation is developed for yield stress $F_{\mathrm{y}}$ of $250 \mathrm{MPa}$. By observing Eq. 5, it can be concluded that both smaller opening ratio $d_{h} / h$ and larger aspect ratio $a / h$ increase the inelastic buckling shear force linearly.

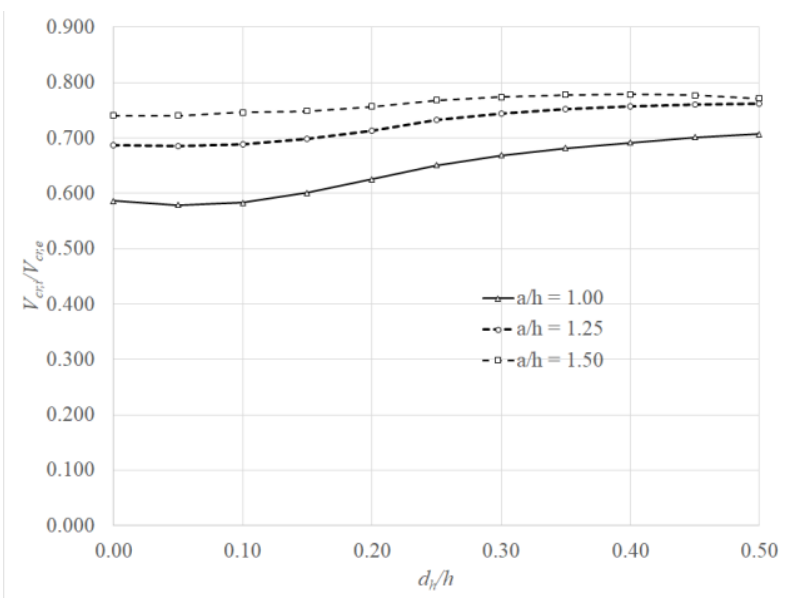

Fig. 14. $V_{c r, \text { inelastic }} / V_{c r, \text { elastic }}$ versus $d_{h} / h$ for various $a / h$.

Table 2. Data used for regression and difference between data and regression equation.

\begin{tabular}{|c|c|c|c|r|}
\hline$d_{h} / h$ & $a / h$ & $V_{c r, i} /\left(V_{c r, e}\right)_{\text {no hole }}$ & $\begin{array}{c}y_{\text {predict }} \\
(\text { Eq. 4) }\end{array}$ & $\begin{array}{c}\text { Differen } \\
\text {-ce }(\%)\end{array}$ \\
\hline$x_{1}$ & $x_{2}$ & $y$ & 0.634 & 8.071 \\
\hline 0.00 & 1.00 & 0.5867 & 0.593 & 4.337 \\
\hline 0.05 & 1.00 & 0.5680 & 0.551 & 2.801 \\
\hline 0.10 & 1.00 & 0.5363 & 0.510 & 1.487 \\
\hline 0.15 & 1.00 & 0.5026 & 0.469 & -0.015 \\
\hline 0.20 & 1.00 & 0.4688 & 0.427 & -1.595 \\
\hline 0.25 & 1.00 & 0.4343 & 0.386 & -1.408 \\
\hline 0.30 & 1.00 & 0.3916 & 0.345 & -0.782 \\
\hline 0.35 & 1.00 & 0.3474 & 0.303 & -0.427 \\
\hline 0.40 & 1.00 & 0.3047 & 0.262 & -1.256 \\
\hline 0.45 & 1.00 & 0.2654 & 0.221 & -3.548 \\
\hline 0.50 & 1.00 & 0.2289 & 0.701 & 1.959 \\
\hline 0.00 & 1.25 & 0.6873 & & \\
\hline
\end{tabular}




\begin{tabular}{|l|l|l|l|r|}
0.05 & 1.25 & 0.6765 & 0.659 & -2.529 \\
\hline 0.10 & 1.25 & 0.6410 & 0.618 & -3.568 \\
\hline 0.15 & 1.25 & 0.6029 & 0.577 & -4.338 \\
\hline 0.20 & 1.25 & 0.5641 & 0.535 & -5.071 \\
\hline 0.25 & 1.25 & 0.5210 & 0.494 & -5.167 \\
\hline 0.30 & 1.25 & 0.4723 & 0.453 & -4.119 \\
\hline 0.35 & 1.25 & 0.4235 & 0.411 & -2.829 \\
\hline 0.40 & 1.25 & 0.3755 & 0.370 & -1.421 \\
\hline 0.45 & 1.25 & 0.3300 & 0.329 & -0.355 \\
\hline 0.50 & 1.25 & 0.2878 & 0.288 & -0.110 \\
\hline 0.00 & 1.50 & 0.7407 & 0.768 & 3.622 \\
\hline 0.05 & 1.50 & 0.7334 & 0.726 & -0.987 \\
\hline 0.10 & 1.50 & 0.7016 & 0.685 & -2.393 \\
\hline 0.15 & 1.50 & 0.6599 & 0.644 & -2.480 \\
\hline 0.20 & 1.50 & 0.6172 & 0.602 & -2.435 \\
\hline 0.25 & 1.50 & 0.5691 & 0.561 & -1.449 \\
\hline 0.30 & 1.50 & 0.5174 & 0.520 & 0.419 \\
\hline 0.35 & 1.50 & 0.4656 & 0.478 & 2.702 \\
\hline 0.40 & 1.50 & 0.4148 & 0.437 & 5.324 \\
\hline 0.45 & 1.50 & 0.3658 & 0.396 & 8.140 \\
\hline 0.50 & 1.50 & 0.3195 & 0.354 & 10.874 \\
\hline
\end{tabular}

\section{Conclusions}

Inelastic buckling behavior of rectangular steel plates with circular openings under shear forces has been studied numerically by employing finite element method. Both large deformation theory and elastic-perfectly plastic material have been utilized in the analyses. It can be concluded that under incremental shear load, yielding of the plate starts at certain parts of the perimeter of the opening and spreads towards the plate edge as the shear force is increased. Furthermore, at buckling shear force level, there is some area in the plate that is still in the elastic range. Both smaller opening ratio $d_{h} / h$ and larger aspect ratio $a / h$ increase the inelastic shear buckling load linearly. Equation to predict buckling loads of a rectangular steel plates with circular opening under shear forces is proposed in this study. Further study is needed if the equation is to be used for conditions beyond the scope of this study.

The authors wish to acknowledge the Faculty of Engineering, Parahyangan Catholic University for the financial support and the Structural Laboratory facility.

\section{References}

1. M. D. V. Real, L.A. Isoldi, A. P. Damas, D. Helbig, Vetor, Rio Grande, 23, 2 (2013)

2. E. Maiorana, C. Pellegrino, C. Modena, Thin-Walled Structures, 47, 3 (2009)

3. M. A. Komur, M. Sonmez, J. of Constructional Res., 112, 54-60 (2015)
4. B. L. Gendy, HBRC J, 12, 296-304 (2016)

5. C. Pellegrino, E. Maiorana, C. Modena, Thin-Walled Structures, 47, 6-7 (2009)

6. C. H. Pham, J. of Constructional Res., 128, 800-811 (2017)

7. C. D. Moen, B. W. Schafer, Thin-Walled Structures, 47, 1597-1607 (2009)

8. C. G. Salmon, J. E. Johnson, F. A. Malhas, Steel Structures Design and Behavior. 5th ed., (Pearson International Edition, USA, 2009)

9. J. H. Ahn, S. Kainuma, W. H. Lee, Y.J. Jeong, and I.T. Kim, Adv. in Material Sci. and Engr., 2016, 6467589 (2016)

10. AASHTO/AWS, Bridge welding code, (American Welding Society, Miami, Fla, USA, 2002) 